\title{
LINEAR REGULARITY FOR A COLLECTION OF SUBSMOOTH SETS IN BANACH SPACES
}

\author{
XI YIN ZHENG ${ }^{\dagger}$ AND KUNG FU NG NG $^{\ddagger}$
}

\begin{abstract}
Using variational analysis, we study the linear regularity for a collection of finitely many closed sets. In particular, we extend duality characterizations of the linear regularity for a collection of finitely many closed convex sets to the possibly nonconvex setting. Moreover the sharpest linear regularity constant can also be dually represented under the subsmoothness assumption.
\end{abstract}

Key words. approximate projection theorem, linear regularity, normal cone, subsmoothness

AMS subject classifications. 90C31, 90C25, 49J52, 46B20

1. Introduction. Linear regularity is a well known notion in mathematical programming and approximation theory. In particular, it plays a key role in establishing a linear convergence rate of iterates generated by the cyclic projection algorithm for finding the projection from a point to the intersection of finitely many closed convex sets (see [3] and references therein).

In this paper, we study the linear regularity of a collection $\left\{A_{1}, \cdots, A_{n}\right\}$ of finitely many closed sets in a Banach space $X$. Here we say that the collection is locally linearly regular at $a \in \bigcap_{i=1}^{n} A_{i}$ (resp. linearly regular) if there exists $\tau \in(0,+\infty)$ such that

$$
\begin{gathered}
d\left(x, \bigcap_{i=1}^{n} A_{i}\right) \leq \tau \sum_{i=1}^{n} d\left(x, A_{i}\right) \text { for all } x \text { close to } a \\
\left(\text { resp. } \quad d\left(x, \bigcap_{i=1}^{n} A_{i}\right) \leq \tau \sum_{i=1}^{n} d\left(x, A_{i}\right) \text { for all } x \in X\right) .
\end{gathered}
$$

The linear regularity has been well studied by many authors in the case when each $A_{i}$ is a closed convex set (see $[2-5,12,18,25]$ and references therein). In 1972, Jameson [12] proved that, for $n=2$, if each $A_{i}$ is a closed convex cone, then $\left\{A_{1}, \cdots, A_{n}\right\}$ is linearly regular if and only if there exists $\tau \in(0,+\infty)$ such that

$$
N\left(\bigcap_{i=1}^{n} A_{i}, 0\right) \cap B_{X^{*}} \subset \tau \sum_{i=1}^{n} N\left(A_{i}, 0\right) \cap B_{X^{*}},
$$

* Research of the first author was supported by the National Natural Science Foundation of P. R. China (Grant No. 10361008) and the Natural Science Foundation of Yunnan Province, China (Grant No. 2003A0002M). The research of second author was supported by an Earmarked Grant from the Research Grant Council of Hong Kong.

${ }^{\dagger}$ Department of Mathematics, Yunnan University, Kunming 650091, P. R. China (xyzheng@ynu.edu.cn).

${ }^{\ddagger}$ Department of Mathematics, The Chinese University of Hong Kong, Shatin, New Territory, Hong Kong (kfng@math.cuhk.edu.hk). 
where $X^{*}$ denotes the dual space of $X$ and $B_{X^{*}}$ denotes the closed unit ball of $X^{*}$. Pang [21] and Lewis and Pang [15] provided necessary conditions for the linear regularity of a collection of finitely many closed convex sets in terms of the normal cone. Afterwards, Bauschke, Borwein and Li [4] established some sufficient conditions in the same line. Recently, it was proved (cf. $[2,20,25]$ ) that if $\left\{A_{1}, \cdots, A_{n}\right\}$ is a collection of closed convex sets in a Banach space $X$ then the following statements are equivalent:

(C1) $\left\{A_{1}, \cdots, A_{n}\right\}$ is linearly regular.

(C2) There exists $\tau \in(0,+\infty)$ such that

$$
N\left(\bigcap_{i=1}^{n} A_{i}, x\right) \cap B_{X^{*}} \subset \tau \sum_{i=1}^{n} N\left(A_{i}, x\right) \cap B_{X^{*}} \text { for all } x \in \bigcap_{i=1}^{n} A_{i} .
$$

(C3) There exists $\tau \in(0,+\infty)$ such that for any $x \in \bigcap_{i=1}^{n} A_{i}$,

$$
N\left(\bigcap_{i=1}^{n} A_{i}, x\right)=\sum_{i=1}^{n} N\left(A_{i}, x\right)
$$

and

$$
\inf \left\{\sum_{i=1}^{n}\left\|x_{i}^{*}\right\|: x_{i}^{*} \in N\left(A_{i}, x\right) \text { and } x^{*}=\sum_{i=1}^{n} x_{i}^{*}\right\} \leq \tau\left\|x^{*}\right\| \quad \forall x^{*} \in N\left(\bigcap_{i=1}^{n} A_{i}, x\right) .
$$

In the terminology of Deutsch, Li and Ward [11], (SC) means that the collection has the strong conical hull intersection property (strong CHIP) at $x$, which is a useful notion for us and has been extensively studied in variational analysis (cf. $[4,5,10,11,16]$ ).

In this paper, we will study the nonconvex case. In view of the fact that a collection $\left\{A_{1}, \cdots, A_{n}\right\}$ of closed convex sets is linearly regular with a constant $\tau$ if and only if $\left\{A_{1}, \cdots, A_{n}\right\}$ is locally linearly regular at each $a \in \operatorname{bd}\left(\bigcap_{i=1}^{n} A_{i}\right)$ with the same constant, it is natural to adopt the local version when one considers a collection of closed sets. While the equivalences among $(\mathrm{C} 1),(\mathrm{C} 2)$ and $(\mathrm{C} 3)$ are not longer valid if one drops the convexity assumption of some $A_{i}$, a natural substitute of convexity in this respect is the subsmoothness, a notion recently introduced and studied by Aussel, Daniilids and Thibault [1], which is a generalization of the well known notion of the prox-regularity (cf. $[6,7,9,23,24]$ and references therein).

In Section 2, we recall some notions in variational analysis and provide some properties of the subsmoothness. In Section 3, as an application of the Ekeland variational principle, we provide a kind of approximate projection result for a closed set, which is very useful for our analysis. In Section 4, in terms of the subsmoothness and the approximate projection result, we establish sufficient and/or necessary conditions for the local linear regularity of a collection of finitely many subsmooth closed sets, which extend the equivalences among $(\mathrm{C} 1),(\mathrm{C} 2)$ and $(\mathrm{C} 3)$ to the nonconvex case. Moreover the constants $\tau$ satisfying (1.1) are represented quantitatively by duality formulas. 
2. Subsmoothness of a closed set. Throughout this paper, we assume that $X$ is a Banach space. Let $A$ be a closed subset of $X$ and $a \in A$.

Let $T_{c}(A, a)$ and $T(A, a)$ denote respectively the Clarke tangent cone and the contingent cone of $A$ at $a$, which are respectively defined by

$$
T_{c}(A, a)=\liminf _{\substack{A \\ x a, t \rightarrow 0^{+}}} \frac{A-x}{t} \text { and } T(A, a)=\limsup _{t \rightarrow 0^{+}} \frac{A-a}{t},
$$

where $x \stackrel{A}{\rightarrow} a$ means that $x \rightarrow a$ with $x \in A$. Thus, $v \in T_{c}(A, a)$ if and only if, for each sequence $\left\{a_{n}\right\}$ in $A$ converging to $a$ and each sequence $\left\{t_{n}\right\}$ in $(0, \infty)$ decreasing to 0 , there exists a sequence $\left\{v_{n}\right\}$ in $X$ converging to $v$ such that $a_{n}+t_{n} v_{n} \in A$ for all $n$, while $v \in T(A, a)$ if and only if there exist a sequence $\left\{v_{n}\right\}$ converging to $v$ and a sequence $\left\{t_{n}\right\}$ in $(0, \infty)$ decreasing to 0 such that $a+t_{n} v_{n} \in A$ for all $n$.

We denote by $N_{c}(A, a)$ the Clarke normal cone of $A$ at $a$, that is,

$$
N_{c}(A, a):=\left\{x^{*} \in X^{*}:\left\langle x^{*}, h\right\rangle \leq 0 \text { for all } h \in T_{c}(A, a)\right\} .
$$

For $\varepsilon \geq 0$ and $a \in A$, the nonempty set

$$
\hat{N}_{\varepsilon}(A, a):=\left\{x^{*} \in X^{*}: \limsup _{x \rightarrow a} \frac{\left\langle x^{*}, x-a\right\rangle}{\|x-a\|} \leq \varepsilon\right\}
$$

is called the set of Fréchet $\varepsilon$-normals of $A$ at $a$. When $\varepsilon=0, \hat{N}_{\varepsilon}(A, a)$ is a convex cone which is called the Fréchet normal cone of $A$ at $a$ and is denoted by $\hat{N}(A, a)$.

Let $N(A, a)$ denote the limiting normal cone of $A$ at $a$, that is,

$$
N(A, a)=\limsup _{\substack{A \rightarrow a, \varepsilon \rightarrow 0^{+} \\ N_{\varepsilon}}} \hat{N}_{\varepsilon, x) .}
$$

Thus, $x^{*} \in N(A, a)$ if and only if there exists a sequence $\left\{\left(x_{n}, \varepsilon_{n}, x_{n}^{*}\right)\right\}$ in $A \times R_{+} \times X^{*}$ such that $\left(x_{n}, \varepsilon_{n}\right) \rightarrow(a, 0), x_{n}^{*} \stackrel{w^{*}}{\rightarrow} x^{*}$ and $x_{n}^{*} \in \hat{N}_{\varepsilon_{n}}\left(A, x_{n}\right)$ for each $n$. It is known that

$$
\hat{N}(A, a) \subset N(A, a) \subset N_{c}(A, a)
$$

(cf. [17] and [18]).

If $A$ is convex, then $T_{c}(A, a)=T(A, a)$ and $N_{c}(A, a)=\hat{N}(A, a)$; in this case,

$$
N_{c}(A, a)=\hat{N}(A, a)=\left\{x^{*} \in X^{*}:\left\langle x^{*}, x\right\rangle \leq\left\langle x^{*}, a\right\rangle \text { for all } x \in A\right\} .
$$

Recall that a Banach space $X$ is called an Asplund space if every continuous convex function on $X$ is Fréchet differentiable at each point of a dense subset of $X$. It is well known (cf. [22]) that $X$ is an Asplund space if and only if every separable subspace of $X$ has a separable dual space. In particular, every reflexive Banach space is an Asplund space. When $X$ is an Asplund space, Mordukhovich and Shao [18] proved that

$$
N_{c}(A, a)=\operatorname{cl}^{*}(\operatorname{co}(N(A, a))) \text { and } N(A, a)=\limsup _{x \rightarrow a} \hat{N}(A, x),
$$


where $\mathrm{cl}^{*}(\cdot)$ denotes the closure with respect to the weak ${ }^{*}$ topology $w^{*}$.

Recall that a closed set $A$ in $X$ is said to be prox-regular at $a \in A$ if there exist $\sigma, r>0$ such that

$$
\left\langle x^{*}-u^{*}, x-u\right\rangle \geq-\sigma\|x-u\|^{2}
$$

whenever $x, u \in A \cap B(a, r), x^{*} \in N_{c}(A, x) \cap B_{X^{*}}$ and $u^{*} \in N_{c}(A, u) \cap B_{X^{*}}$. Readers can find some interesting properties of the prox-regularity in [23] and [24].

As a generalization of the prox-regularity, Aussel, Daniilidis and Thibault [1] introduced and studied the subsmoothness. A closed set $A$ in $X$ is said to be subsmooth at $a \in A$ if for any $\varepsilon>0$ there exists $r>0$ such that

$$
\left\langle x^{*}-u^{*}, x-u\right\rangle \geq-\varepsilon\|x-u\|
$$

whenever $x, u \in A \cap B(a, r), x^{*} \in N_{c}(A, x) \cap B_{X^{*}}$ and $u^{*} \in N_{c}(A, u) \cap B_{X^{*}}$.

Taking $x^{*}=0,(2.2)$ reduces to $\left\langle u^{*}, x-u\right\rangle \leq \varepsilon\|x-u\|$. On the other hand, noting that $\left\langle x^{*}-u^{*}, x-u\right\rangle \geq-2 \varepsilon\|x-u\|$ if $\left\langle x^{*}, u-x\right\rangle \leq \varepsilon\|x-u\|$ and $\left\langle u^{*}, x-u\right\rangle \leq \varepsilon\|x-u\|$, it follows that $A$ is subsmooth at $a \in A$ if and only if for any $\varepsilon>0$ there exists $r>0$ such that

$$
\left\langle u^{*}, x-u\right\rangle \leq \varepsilon\|x-u\| \text { for all } x \in A \cap B(u, r)
$$

whenever $u \in A \cap B(a, r)$ and $u^{*} \in N_{c}(A, u) \cap B_{X^{*}}$. Thus, for any $\varepsilon>0$ there exists $r>0$ such that

$$
N_{c}(A, u) \subset \hat{N}_{\varepsilon}(A, u) \text { for all } u \in A \cap B(a, r)
$$

provided that $A$ is subsmooth at $a$. Hence

$$
\text { subsmoothness of } A \text { at } a \Longrightarrow N_{c}(A, a)=\hat{N}(A, a) \text {. }
$$

Usually, $A$ is said to be regular at $a$ in the Clarke sense if $N_{c}(A, a)=\hat{N}(A, a)$.

It is known (cf. [17, Corollary 1.96]) that $\hat{N}(A, u) \cap B_{X^{*}}=\hat{\partial} d(\cdot, A)(u)$ for $u \in A$, where $\hat{\partial}$ denotes the Fréchet subdifferential. Hence $x^{*} \in \hat{N}(A, u) \cap B_{X^{*}}$ if and only if for any $\varepsilon>0$ there exists $r>0$ such that

$$
\left\langle x^{*}, x-u\right\rangle \leq d(x, A)+\varepsilon\|x-u\| \quad \forall x \in B(u, r) .
$$

The following proposition shows that a strengthened condition similar to (2.5) provides a characterization of the subsmoothness. As the result will be quoted several times we include its simple proof here.

Proposition 2.1. Let $A$ be a closed subset of $X$. Then $A$ is subsmooth at $a \in A$ if and only if for any $\varepsilon>0$ there exists $r>0$ such that

$$
\left\langle u^{*}, x-u\right\rangle \leq d(x, A)+\varepsilon\|x-u\| \text { for all } x \in B(a, r)
$$

whenever $u \in A \cap B(a, r)$ and $u^{*} \in N_{c}(A, u) \cap B_{X^{*}}$. 
Proof. Since $d(x, A)=0$ for all $x \in A,(2.6)$ implies (2.3). Hence, the sufficiency part holds. Conversely, suppose that $A$ is subsmooth at $a \in A$. Let $\varepsilon>0$ and take $r>0$ such that

$$
\left\langle u^{*}, z-u\right\rangle \leq \frac{\varepsilon}{2}\|z-u\| \text { for all } z \in A \cap B(a, 2 r)
$$

whenever $u \in A \cap B(a, r)$ and $u^{*} \in N_{c}(A, u) \cap B_{X^{*}}$. Let $x \in B(a, r), u \in A \cap B(a, r)$ and $u^{*} \in N_{c}(A, u) \cap B_{X^{*}}$. Then $d(x, A) \leq\|x-a\|<r$. Thus, there exists a sequence $\left\{u_{n}\right\}$ in $A \cap B(x, r)$ such that $\left\|x-u_{n}\right\| \rightarrow d(x, A)$. Hence $\left\|u_{n}-a\right\| \leq\left\|u_{n}-x\right\|+\|x-a\|<2 r$. It follows from (2.7) that

$$
\begin{aligned}
\left\langle u^{*}, x-u\right\rangle & =\left\langle u^{*}, x-u_{n}\right\rangle+\left\langle u^{*}, u_{n}-u\right\rangle \\
& \leq\left\|x-u_{n}\right\|+\frac{\varepsilon}{2}\left\|u_{n}-u\right\| \\
& \leq\left\|x-u_{n}\right\|+\frac{\varepsilon}{2}\left(\left\|u_{n}-x\right\|+\|x-u\|\right) .
\end{aligned}
$$

Letting $n \rightarrow \infty$, one has

$$
\left\langle u^{*}, x-u\right\rangle \leq d(x, A)+\frac{\varepsilon}{2}(d(x, A)+\|x-u\|) \leq d(x, A)+\varepsilon\|x-u\| .
$$

This shows that the necessity part holds. The proof is completed.

Proposition 2.2. Let $X, Y$ be Banach spaces, $\Omega$ a closed convex subset of $Y$ and $g: X \rightarrow Y$ a continuously differentiable function. Let $a \in g^{-1}(\Omega)$ and suppose that $g^{\prime}(a)$ is surjective, where $g^{\prime}(a)$ denotes the derivative of $g$ at $a$. Then there exists a neighborhood $U$ of a such that the following statements hold.

(i) $N_{c}\left(g^{-1}(\Omega), u\right)=\left(g^{\prime}(u)\right)^{*}(N(\Omega, g(u)))$ for any $u \in g^{-1}(\Omega) \cap U$, where $\left(g^{\prime}(u)\right)^{*}$ denotes the adjoint operator of $g^{\prime}(u)$.

(ii) $g^{-1}(\Omega)$ is subsmooth at each point of $g^{-1}(\Omega) \cap U$.

Proof. Since $g^{\prime}(a)$ is surjective, there exists $l>0$ such that $2 l B_{Y} \subset g^{\prime}(a)\left(B_{X}\right)$. Since $x \mapsto g^{\prime}(x)$ is continuous, it follows that there exists $r>0$ such that

$$
l B_{Y} \subset g^{\prime}(x)\left(B_{X}\right) \text { for all } x \in B(a, r) .
$$

To prove (i), it suffices to show the inclusion

$$
N_{c}\left(g^{-1}(\Omega), u\right) \subset g^{\prime}(u)^{*}(N(\Omega, g(u))) \quad \forall u \in g^{-1}(\Omega) \cap B(a, r)
$$

(the converse inclusion follows easily from [17, Theorem 1.17]). Suppose to the contrary that there exists $u \in g^{-1}(\Omega) \cap B(a, r)$ such that

$$
x^{*} \in N_{c}\left(g^{-1}(\Omega), u\right) \backslash g^{\prime}(u)^{*}(N(\Omega, g(u))) .
$$

Since the adjoint operator $g^{\prime}(u)^{*}$ is weak ${ }^{*}$-weak ${ }^{*}$ continuous and $g^{\prime}(u)$ is surjective (by $(2.8)), g^{\prime}(u)^{*}(N(\Omega, g(u))) \cap B_{X^{*}}$ is weak ${ }^{*}$ closed. This and the Krein-Smulian 
theorem imply that $g^{\prime}(u)^{*}(N(\Omega, g(u)))$ is weak ${ }^{*}$ closed. By (2.10) and the separation theorem, there exists $h_{0} \in X$ such that

$$
\begin{aligned}
\left\langle x^{*}, h_{0}\right\rangle & >\sup \left\{\left\langle g^{\prime}(u)^{*}\left(y^{*}\right), h_{0}\right\rangle: y^{*} \in N(\Omega, g(u))\right\} \\
& =\sup \left\{\left\langle y^{*}, g^{\prime}(u)\left(h_{0}\right)\right\rangle: y^{*} \in N(\Omega, g(u))\right\} .
\end{aligned}
$$

It follows from the convexity of $\Omega$ that $\left\langle x^{*}, h_{0}\right\rangle>0$ and $g^{\prime}(u)\left(h_{0}\right) \in T_{c}(\Omega, g(u))$. Take an arbitrary sequence $\left\{x_{n}\right\}$ in $g^{-1}(\Omega)$ converging to $u$ and an arbitrary sequence $\left\{t_{n}\right\}$ in $(0,+\infty)$ decreasing to 0 . Then $g\left(x_{n}\right) \stackrel{\Omega}{\rightarrow} g(u)$ and hence there exists a sequence $y_{n} \rightarrow g^{\prime}(u)\left(h_{0}\right)$ such that $g\left(x_{n}\right)+t_{n} y_{n} \in \Omega$ for all $n$. Since $g$ is continuously differentiable, (2.8) and the Lyusternik-Graves theorem (cf. [17, Theorem 1.57]) imply that

$$
d\left(x_{n}+t_{n} h_{0}, g^{-1}\left(g\left(x_{n}\right)+t_{n} y_{n}\right)\right) \leq L\left\|g\left(x_{n}+t_{n} h_{0}\right)-g\left(x_{n}\right)-t_{n} y_{n}\right\|
$$

for some $L \in(0,+\infty)$ and all $n$ large enough. Noting that

$$
g\left(x_{n}+t_{n} h_{0}\right)-g\left(x_{n}\right)=g^{\prime}(u)\left(t_{n} h_{0}\right)+o\left(t_{n}\right),
$$

it follows that for each $n$ large enough there exists $\tilde{x}_{n} \in X$ such that

$$
\tilde{x}_{n} \in g^{-1}\left(g\left(x_{n}\right)+t_{n} y_{n}\right) \subset g^{-1}(\Omega)
$$

and

$$
\left\|x_{n}+t_{n} h_{0}-\tilde{x}_{n}\right\| \leq 2 L\left(t_{n}\left\|g^{\prime}(u)\left(h_{0}\right)-y_{n}\right\|+\left\|o\left(t_{n}\right)\right\|\right) .
$$

This and $y_{n} \rightarrow g^{\prime}(u)\left(h_{0}\right)$ imply that $h_{n}:=\frac{\tilde{x}_{n}-x_{n}}{t_{n}} \rightarrow h_{0}$ and $x_{n}+t_{n} h_{n}=\tilde{x}_{n} \in g^{-1}(\Omega)$. This shows that $h_{0} \in T_{c}\left(g^{-1}(\Omega), u\right)$, which is not possible because $x^{*} \in N_{c}\left(g^{-1}(\Omega), u\right)$ and $\left\langle x^{*}, h_{0}\right\rangle>0$. Hence, (2.9) holds and hence (i) is shown.

Next we show that (ii) holds. Let $z \in g^{-1}(\Omega) \cap B\left(a, \frac{r}{2}\right)$ and $\varepsilon>0$. Then there exists $\delta \in\left(0, \frac{r}{2}\right)$ such that

$$
\left\|g^{\prime}\left(u_{1}\right)-g^{\prime}\left(u_{2}\right)\right\|<\frac{l \varepsilon}{2} \text { for any } u_{1}, u_{2} \in B(z, 2 \delta) .
$$

Let $u \in g^{-1}(\Omega) \cap B(z, \delta)$ and $u^{*} \in N_{c}\left(g^{-1}(\Omega), u\right) \cap B_{X^{*}}$. Then, by (i), there exists $y^{*} \in N(\Omega, g(u))$ such that $u^{*}=\left(g^{\prime}(u)\right)^{*}\left(y^{*}\right)$. Take $y \in B_{Y}$ such that $\left\|y^{*}\right\| \leq 2\left\langle y^{*}, y\right\rangle$. By (2.8), there exists $v \in B_{X}$ such that $l y=g^{\prime}(u)(v)$. Hence,

$$
l\left\|y^{*}\right\| \leq 2\left\langle y^{*}, g^{\prime}(u)(v)\right\rangle=2\left\langle u^{*}, v\right\rangle \leq 2 .
$$

By the convexity of $\Omega$, one has

$$
\left\langle y^{*}, g(x)-g(u)\right\rangle \leq 0 \text { for all } x \in g^{-1}(\Omega) .
$$

Noting that

$$
\left\langle u^{*}, x-u\right\rangle=\left\langle\left(g^{\prime}(u)\right)^{*}\left(y^{*}\right), x-u\right\rangle=\left\langle y^{*}, g^{\prime}(u)(x-u)\right\rangle,
$$


it follows that for any $x \in g^{-1}(\Omega) \cap B(u, \delta)$,

$$
\begin{aligned}
\left\langle u^{*}, x-u\right\rangle & \leq\left\langle y^{*}, g^{\prime}(u)(x-u)-(g(x)-g(u))\right\rangle \\
& \leq \frac{2}{l}\left\|g(x)-g(u)-g^{\prime}(u)(x-u)\right\| \\
& \leq \frac{2}{l}\left\|g^{\prime}(u+\theta(x-u))-g^{\prime}(u)\right\|\|x-u\|
\end{aligned}
$$

where $\theta \in(0,1)$. Since $\|u+\theta(x-u)-z\| \leq\|u-z\|+\theta\|x-u\|<2 \delta$, it follows from (2.12) that

$$
\left\langle u^{*}, x-u\right\rangle \leq \varepsilon\|x-u\| \text { for any } x \in g^{-1}(\Omega) \cap B(z, \delta) .
$$

Therefore, $g^{-1}(\Omega)$ is subsmooth at $z$. This shows that (ii) holds.

Remark. It is known (see [17, Theorem 1.17]) that if $g$ is strictly differentiable at $a \in g^{-1}(\Omega)$ such that $g^{\prime}(a)$ is surjective then

$$
N\left(g^{-1}(\Omega), a\right)=\left(g^{\prime}(a)\right)^{*}(N(\Omega, g(a))) ;
$$

noting that $\left(g^{\prime}(a)\right)^{*}(N(\Omega, g(a)))$ is weak ${ }^{*}$ closed and convex, it follows from (2.1) that

$$
N_{c}\left(g^{-1}(\Omega), a\right)=\left(g^{\prime}(a)\right)^{*}(N(\Omega, g(a)))
$$

when $X$ is an Asplund space. Hence (i) of Proposition 2.2 is a consequence of [17, Theorem 1.17] when $X$ is an Asplund space.

It is also known (cf. [8, P.108, Corollary 1]) that if $g$ is strictly differentiable at $a$ with $g^{\prime}(a)(X) \cap \operatorname{int}(T(\Omega, g(a)) \neq \emptyset$ and if $\Omega$ admits a hypertangent vector at $g(a)$, namely there exist $v \in Y$ and $r>0$ such that

$$
\Omega \cap B(g(a), r)+t B(v, r) \subset \Omega \text { for all } t \in(0, r),
$$

then $N_{c}\left(g^{-1}(\Omega), a\right)=\left(g^{\prime}(a)\right)^{*}(N(\Omega, g(a)))$. In contrast, Proposition 2.2 does not require that $\Omega$ admits a hypertangent vector at $g(a)$.

The following Proposition 2.3 demonstrates an interesting fact that, in an Asplund space, the subsmoothness on an open subset of $A$ can be described in terms of the Fréchet normal cone (rather than the Clarke normal cone). To do this, we need the following lemma, which is also used in Section 4.

Lemma 2.1. Let $A$ be a closed subset of $X$ and $a \in A$. Suppose that for any $\varepsilon>0$ there exists $r>0$ such that

$$
\left\langle u^{*}, x-a\right\rangle \leq \varepsilon\|x-a\| \quad \forall x \in A \cap B(a, r) \text { and } \forall u^{*} \in \hat{N}(A, a) \cap B_{X^{*}} .
$$

Then $\hat{N}(A, a)$ is weak ${ }^{*}$ closed.

Proof. Let $\varepsilon$ be an arbitrary number in $(0,+\infty)$ and take $r>0$ such that (2.13) holds. Since $\hat{N}(A, a)$ is convex, by the Krein-Smulian theorem it suffices to 
show that $\hat{N}(A, a) \cap B_{X^{*}}$ is weak ${ }^{*}$ closed. Let $\left\{u_{j}^{*}\right\}$ be a net in $\hat{N}(A, a) \cap B_{X^{*}}$ convergent to $x^{*} \in X^{*}$ with respect to the weak ${ }^{*}$ topology. Then, $x^{*} \in B_{X^{*}}$ (because $B_{X^{*}}$ is weak ${ }^{*}$ closed) and $\left\langle u_{j}^{*}, x\right\rangle \rightarrow\left\langle x^{*}, x\right\rangle$ for all $x \in X$. It follows from (2.13) that $\left\langle x^{*}, x-a\right\rangle \leq \varepsilon\|x-a\|$ for all $x \in A \cap B(a, r)$. This and the arbitrariness of $\varepsilon$ imply that $x^{*} \in \hat{N}(A, a)$. Hence $x^{*} \in \hat{N}(A, a) \cap B_{X^{*}}$. This shows that $\hat{N}(A, a) \cap B_{X^{*}}$ is weak ${ }^{*}$ closed. The proof is completed.

Proposition 2.3. Let $A$ be a closed subset of $X$ and $U$ is an open subset of $X$. Suppose that $X$ is an Asplund space. Then $A$ is subsmooth at each point of $A \cap U$ if and only if for any $z \in A \cap U$ and $\varepsilon>0$ there exists $r>0$ such that (2.3) holds whenever $u \in \operatorname{bd}(A) \cap B(z, r)$ and $u^{*} \in \hat{N}(A, u) \cap B_{X^{*}}$.

Proof. Since $\hat{N}(A, x) \subset N_{c}(A, x)$ for all $x \in A$, the necessity part is clear. For the sufficiency part, we need only show that $N_{c}(A, z)=\hat{N}(A, z)$ for any $z \in A \cap U$. Let $z \in A \cap U$ and $\varepsilon>0$. Take $r>0$ such that (2.3) holds for any $u \in B(z, r) \cap \operatorname{bd}(A)$ and $u^{*} \in \hat{N}(A, u) \cap B_{X^{*}}$. Let $z^{*} \in N(A, z)$. Since $X$ is an Asplund space, there exists a sequence $\left\{\left(u_{n}, u_{n}^{*}\right)\right\}$ in $X \times X^{*}$ such that

$$
u_{n} \stackrel{A}{\rightarrow} z, u_{n}^{*} \stackrel{w^{*}}{\rightarrow} z^{*} \text { and } u_{n}^{*} \in \hat{N}\left(A, u_{n}\right) .
$$

Hence, there exists $M \in(0,+\infty)$ such that each $\left\|u_{n}^{*}\right\|<M$. Without loss of generality, we can assume that $u_{n} \in B(z, r)$. It follows from (2.3) that

$$
\left\langle\frac{u_{n}^{*}}{M}, x-u_{n}\right\rangle \leq \varepsilon\left\|x-u_{n}\right\| \text { for all } x \in B\left(u_{n}, r\right) \cap A .
$$

Letting $n \rightarrow \infty$, one has

$$
\left\langle\frac{z^{*}}{M}, x-z\right\rangle \leq \varepsilon\|x-z\| \text { for all } x \in B(z, r) \cap A .
$$

It follows that $z^{*} \in \hat{N}(A, z)$. Hence, $N(A, z) \subset \hat{N}(A, z)$. Since the converse inclusion automatically holds, $N(A, z)=\hat{N}(A, z)$. Since $X$ is an Asplund space, it follows from (2.1) that $N_{c}(A, z)=\mathrm{cl}^{*}(\operatorname{co}(\hat{N}(A, z)))$. Noting that $\hat{N}(A, z)$ is a convex cone, this means that $N_{c}(A, z)=\mathrm{cl}^{*}(\hat{N}(A, z))$. It follows from Lemma 2.1 that $N_{c}(A, z)=\hat{N}(A, z)$. The proof is completed.

A natural question is : Can the open set $U$ in Proposition 2.3 be replaced by a singleton $\{a\}$ with $a \in A$ ? That is, is $A$ subsmooth at a given point $a$ of $A$ if for any $\varepsilon>0$ there exists $r>0$ such that (2.3) holds whenever $u \in \operatorname{bd}(A) \cap B(a, r)$ and $u^{*} \in \hat{N}(A, u) \cap B_{X^{*}}$ ?

While we don't have an answer for this question, we have the following result:

Proposition 2.4. Let $X$ be an Asplund space, A a closed subset of $X$ and $a \in A$. Then the following two statements are equivalent.

(i) For any $\varepsilon>0$ there exists $r>0$ such that (2.3) holds whenever $u \in A \cap B(a, r)$ and $u^{*} \in N(A, u) \cap B_{X^{*}}$. 
(ii) same as (i) but the limiting normal cone $N(A, \cdot)$ being replaced by the Fréchet normal cone $\hat{N}(A, \cdot)$.

Proof. (i) $\Rightarrow$ (ii) is trivial. To prove (ii) $\Rightarrow$ (i), let $\varepsilon>0$ and take $r>0$ such that (2.3) holds whenever $u \in \operatorname{bd}(A) \cap B(a, r))$ and $u^{*} \in \hat{N}(A, u) \cap B_{X^{*}}$. Let $u \in A \cap B(a, r)$ and $x^{*} \in N(A, u) \cap B_{X^{*}}$. Since $X$ is an Asplund space, (2.1) implies that there exists a sequence $\left\{\left(u_{k}, u_{k}^{*}\right)\right\} \subset(\operatorname{bd}(A) \cap B(a, r)) \times X^{*}$ such that

$$
u_{k} \rightarrow u, u_{k}^{*} \stackrel{w^{*}}{\rightarrow} u^{*} \text { and } u_{k}^{*} \in \hat{N}\left(A, u_{k}\right) .
$$

It follows from (2.3) that $\left\langle u_{k}^{*}, x-u_{k}\right\rangle \leq \varepsilon\left\|x-u_{k}\right\|$ for all $x \in A \cap B\left(u_{k}, r\right)$ and all $k$. Letting $k \rightarrow \infty$, one has $\left\langle u^{*}, x-u\right\rangle \leq \varepsilon\|x-u\|$ for all $x \in A \cap B(u, r)$. This shows that (i) holds. The proof is completed.

Corollary 2.1. Let $X$ be an Asplund space, $A$ a closed subset of $X$ and $a \in$ $A$. Suppose that for any $\varepsilon>0$ there exists $r>0$ such that (2.3) holds whenever $u \in \operatorname{bd}(A) \cap B(a, r)$ and $u^{*} \in \hat{N}(A, u) \cap B_{X^{*}}$. Then $A$ is regular at $a$ in the sense of Clarke.

Proof. By Proposition 2.4, N(A,a)= $\hat{N}(A, a)$. It follows from (2.1) that $N_{c}(A, a)=\operatorname{cl}^{*}(\operatorname{co}(\hat{N}(A, a)))=\operatorname{cl}^{*}(\hat{N}(A, a))$. This and Lemma 2.1 show that $A$ is regular at $a$ in the sense of Clarke. The proof is completed.

3. Approximate projection theorem in Banach spaces. Using BronsteadRockafellar theorem, it was proved in [19] that if $A$ is a closed convex nonempty subset of a Banach space $X$ and $x \in X \backslash A$ then for any $\gamma \in(0,1)$ there exist $a \in \operatorname{bd}(A)$ and $a^{*} \in N(A, a)$ with $\left\|a^{*}\right\|=1$ such that

$$
\gamma\|x-a\|<\min \left\{d(x, A),\left\langle a^{*}, x-a\right\rangle\right\} .
$$

By virtue of the well known Ekeland variational principle, we provide below a nonconvex generalization of the above projection result which will play as a key tool in the proofs of our main results in Section 4.

In order to present our results in a unified manner, we use the notion of a prenormal structure in this section. We say that $\tilde{N}$ is a prenormal structure on $X$ if for any nonempty closed subset $A$ of $X, \tilde{N}(A, \cdot): A \rightrightarrows X^{*}$ is a multifunction of cone values such that the following properties hold:

(P1) If a continuous convex function $f$ on $X$ attains its global minimum over $A$ at $\bar{x} \in A$ then for any $\varepsilon>0$ there exist $x_{1}, x_{2} \in B(\bar{x}, \varepsilon)$ such that

$$
x_{2} \in A \text { and } 0 \in \partial f\left(x_{1}\right)+\tilde{N}\left(A, x_{2}\right)+\varepsilon B_{X^{*}},
$$

where $\partial f\left(x_{1}\right)$ is the subdifferential of $f$ at $x_{1}$ in the convex analysis sense.

(P2) $\tilde{N}(A, a)=\{0\}$ for any $a \in \operatorname{int}(A)$.

It is known that the Clarke normal structure is a prenormal structure on every Banach space and the Fréchet normal structure is also a prenormal structure on 
every Asplund space. Various abstract prenormal structures, with generally different properties, have been used by many authors (for the details see Mordukhovich's recent book [17, Sections 2.5.1 and 2.6.9] and references therein).

Theorem 3.1. Let $X$ be a Banach space and $\tilde{N}$ be a prenormal structure on $X$. Let $A$ be a closed nonempty subset of $X$. Let $\gamma \in(0,1)$. Then for any $x \notin A$ there exist $a \in \operatorname{bd}(A)$ and $a^{*} \in \tilde{N}(A, a)$ with $\left\|a^{*}\right\|=1$ such that (3.1) holds.

Proof. Let $x \in X \backslash A$. Then $d(x, A)>0$. Let $\varepsilon \in(0,1)$ be small enough such that

$$
\varepsilon<\min \left\{\frac{\left(1-\gamma^{\frac{1}{2}}\right) d(x, A)}{2 \gamma^{\frac{1}{2}}}, \frac{\left(1-\gamma^{\frac{1}{2}}\right) d(x, A)}{4+\left(2+2 \gamma^{\frac{1}{2}}\right) d(x, A)}\right\}
$$

and take $z_{0} \in A$ such that $\left\|z_{0}-z\right\|<d(x, A)+\varepsilon$. Let $\phi(z):=\|z-x\|+\delta_{A}(z)$ for all $z \in X$. Then $\phi$ is a proper lower semicontinuous function and $\phi\left(z_{0}\right)<\inf _{z \in X} \phi(z)+\varepsilon$. By the Ekeland variational principle, there exists $\bar{z} \in A$ such that $\phi(\bar{z}) \leq \phi\left(z_{0}\right)$ and $\phi(\bar{z}) \leq \phi(z)+\varepsilon\|z-\bar{z}\|$ for all $z \in X$. Hence

$$
\|\bar{z}-x\|<d(x, A)+\varepsilon
$$

and the continuous convex function $f(z):=\|z-x\|+\varepsilon\|z-\bar{z}\|$ attains its global minimum over $A$ at $\bar{z}$. It follows from (P1) that there exist $z_{1}, a \in B(\bar{z}, \varepsilon)$ such that

$$
a \in A \text { and } 0 \in \partial f\left(z_{1}\right)+\tilde{N}(A, a)+\varepsilon B_{X^{*}} .
$$

Since $z_{1} \neq x$ (by $x \notin A, \bar{z} \in A$ and $\varepsilon<d(x, A)$ ), there exist $z_{1}^{*}, z_{2}^{*} \in X^{*}$ such that

$$
\left\|z_{1}^{*}\right\|=1,\left\|z_{2}^{*}\right\|<2 \varepsilon,\left\langle z_{1}^{*}, z_{1}-x\right\rangle=\left\|z_{1}-x\right\| \text { and }-z_{1}^{*}+z_{2}^{*} \in \tilde{N}(A, a) .
$$

It follows from (3.3) that $\|x-a\|<d(x, A)+2 \varepsilon$. This and (3.2) imply that

$$
\gamma^{\frac{1}{2}}\|x-a\|<d(x, A) .
$$

Let $a^{*}:=\frac{-z_{1}^{*}+z_{2}^{*}}{\left\|-z_{1}^{*}+z_{2}^{*}\right\|}$. Then, $a^{*} \in \tilde{N}(A, a)$ and so $a \in \operatorname{bd}(A)$ (by (P2)). Note that

$$
\begin{aligned}
\left\langle a^{*}, x-a\right\rangle & =\frac{\left\langle z_{1}^{*}, z_{1}-x\right\rangle+\left\langle z_{1}^{*}, a-z_{1}\right\rangle+\left\langle z_{2}^{*}, x-a\right\rangle}{\left\|-z_{1}^{*}+z_{2}^{*}\right\|} \\
& \geq \frac{\left\|z_{1}-x\right\|-2 \varepsilon-2 \varepsilon\|x-a\|}{1+2 \varepsilon} \\
& \geq \frac{(1-2 \varepsilon)\|x-a\|-4 \varepsilon}{1+2 \varepsilon} \\
& \geq \frac{(1-2 \varepsilon) d(x, A)-4 \varepsilon}{1+2 \varepsilon} .
\end{aligned}
$$

This and (3.2) imply that $\gamma^{\frac{1}{2}} d(x, A)<\left\langle a^{*}, x-a\right\rangle$. It follows from (3.4) and $\gamma \in(0,1)$ that (3.1) holds. The proof is completed. 
When $A$ is a closed convex nonempty set, the property $N(A, a) \neq\{0\}$ means that $a$ is a support point of $A$; thus the following corollary of Theorem 3.1 may be viewed as a nonconvex generalization of the Bishop-Phelps density theorem (cf. [22]).

Corollary 3.1. Let $A$ be a closed nonempty subset of a Banach space $X$ and $\tilde{N}$ be a prenormal structure on $X$. Let $D:=\{a \in \operatorname{bd}(A): \tilde{N}(A, a) \neq\{0\}\}$. Then $D$ is a dense subset of $\mathrm{bd}(A)$.

Proof. Let $\bar{x} \in \operatorname{bd}(A)$ and $\varepsilon>0$. Then there exists $x \notin A$ such that $\|x-\bar{x}\|<\frac{\varepsilon}{4}$. By Theorem 3.1 there exists $a \in D$ such that $\frac{1}{2}\|x-a\|<d(x, A)<\frac{\varepsilon}{4}$, and so $\|x-a\|<\frac{\varepsilon}{2}$. Therefore, $\|\bar{x}-a\|<\varepsilon$. The proof is completed.

When $X$ is an Asplund space and $\tilde{N}$ is the Fréchet normal structure, Corollary 3.1 was proved by Mordukhivich and Shao [18].

Remark. In general, in Theorem 3.1 one cannot take $\gamma=1$ even when $\tilde{N}$ is the Clarke normal structure and $A$ is a closed convex set. For example, let $X$ be a nonreflexive Banach space. Then, by the James theorem there exists $x^{*} \in X^{*}$ with $\left\|x^{*}\right\|=1$ such that

$$
\left\langle x^{*}, z\right\rangle<1 \quad \forall z \in B_{X} .
$$

Let $A:=\left\{x \in X:\left\langle x^{*}, x\right\rangle \leq 1\right\}$. We claim that $\left\langle a^{*}, x-a\right\rangle<\|x-a\|$ for any $x \in X \backslash A$, any $a \in A$ and any $a^{*} \in N(A, a)$ with $\left\|a^{*}\right\|=1$. Indeed, suppose to the contrary that there exist $x_{0} \in X \backslash A, a_{0} \in A$ and $a_{0}^{*} \in N\left(A, a_{0}\right)$ with $\left\|a_{0}^{*}\right\|=1$ such that $\left\langle a_{0}^{*}, x_{0}-a_{0}\right\rangle=\left\|x_{0}-a_{0}\right\|$. Then $a_{0} \in \operatorname{bd}(A)$, that is, $\left\langle x^{*}, a_{0}\right\rangle=1$. By the definition of $A$, it is clear that $N\left(A, a_{0}\right)=R_{+} x^{*}$ and so $a_{0}^{*}=x^{*}$. Hence $\left\langle x^{*}, \frac{x_{0}-a_{0}}{\left\|x_{0}-a_{0}\right\|}\right\rangle=1$, contradicting (3.5).

In the next section, we will apply Theorem 3.1 to the following two cases:

(BC) $X$ is a general Banach space and $\tilde{N}(A, a)=N_{c}(A, a)$ for any closed subset $A$ of $X$ and $a \in A$.

(AF) $X$ is an Asplund space and $\tilde{N}(A, a)=\hat{N}(A, a)$ for any closed subset $A$ of $X$ and $a \in A$.

4. Main results. In this section, we establish some relationships concerning the local linear regularity of a collection of closed sets in a Banach space. First we provide a relationship between the local linear regularity and the linear regularity for a collection of finitely many closed convex sets.

Proposition 4.1. Let $X$ be a Banach space and $C_{1}, \cdots, C_{n}$ be closed convex subsets of $X$ such that $\bigcap_{i=1}^{n} C_{i} \neq \emptyset$. Then $\left\{C_{1}, \cdots, C_{n}\right\}$ is linearly regular if and only if there exists $\tau \in(0,+\infty)$ such that $\left\{C_{1}, \cdots, C_{n}\right\}$ is locally linearly regular at each point of $\operatorname{bd}\left(\bigcap_{i=1}^{n} C_{i}\right)$ with the same constant $\tau$. 
Proof. The necessity is trivial. To prove the sufficiency, let $x \in X \backslash \bigcap_{i=1}^{n} C_{i}$ and $\gamma \in(0,1)$. Then, by Theorem 3.1 (applied to case $(\mathrm{BC}))$ there exist $a \in \operatorname{bd}\left(\bigcap_{i=1}^{n} C_{i}\right)$ and $a^{*} \in N\left(\bigcap_{i=1}^{n} C_{i}, a\right)$ with $\left\|a^{*}\right\|=1$ such that

$$
\gamma\|x-a\| \leq\left\langle a^{*}, x-a\right\rangle .
$$

Letting $L_{a}:=\left\{z \in X:\left\langle a^{*}, z\right\rangle \leq\left\langle a^{*}, a\right\rangle\right\}$, it follows from the convexity of each $C_{i}$ that $\bigcap_{i=1}^{n} C_{i} \subset L_{a}$. Hence

$$
d\left(z, L_{a}\right) \leq d\left(z, \bigcap_{i=1}^{n} C_{i}\right) \text { for all } z \in X .
$$

From $\left\|a^{*}\right\|=1$, it is easy to verify that $d\left(z, L_{a}\right)=\left\langle a^{*}, z-a\right\rangle$ for all $z \in X \backslash L_{a}$. Applying this to $z:=a+t(x-a)$, it follows from (4.1) and (4.2) that

$$
\gamma t\|x-a\| \leq d\left(a+t(x-a), \bigcap_{i=1}^{n} C_{i}\right) \text { for all } t>0 .
$$

Since $\left\{C_{1}, \cdots, C_{n}\right\}$ is locally linearly regular at $a$ with the constant $\tau$, there exists $t \in(0,1)$ small enough such that

$$
d\left(a+t(x-a), \bigcap_{i=1}^{n} C_{i}\right) \leq \tau \sum_{i=1}^{n} d\left(a+t(x-a), C_{i}\right) .
$$

By the convexity of $C_{i}$ and $a \in C_{i}$, one has

$$
d\left(a+t(x-a), C_{i}\right) \leq t d\left(x, C_{i}\right) .
$$

It follows from (4.3) and (4.4) that

$$
\gamma d\left(x, \bigcap_{i=1}^{n} C_{i}\right) \leq \gamma\|x-a\| \leq \tau \sum_{i=1}^{n} d\left(x, C_{i}\right) .
$$

Letting $\gamma \rightarrow 1$, one has $d\left(x, \bigcap_{i=1}^{n} C_{i}\right) \leq \tau \sum_{i=1}^{n} d\left(x, C_{i}\right)$. This completes the proof.

In view of Proposition 4.1, we see that it is pertinent to study the local linear regularity for a collection of nonconvex closed sets. In the remainder of this section, we always assume that $X$ is a Banach space (unless otherwise stated) and that $\left\{A_{1}, \cdots, A_{n}\right\}$ is a collection of closed sets in $X$ with intersection $A$ containing $a$ :

$$
a \in A:=\bigcap_{i=1}^{n} A_{i} .
$$

The modulus of the linear regularity of the collection $\left\{A_{1}, \cdots, A_{n}\right\}$ at $a \in A$ is denoted by $\eta\left(A_{1}, \cdots, A_{n} ; a\right)$ and defined by

$$
\eta\left(A_{1}, \cdots, A_{n} ; a\right):=\inf \{\tau>0:(1.1) \text { holds }\} .
$$


Thus, $\eta\left(A_{1}, \cdots, A_{n} ; a\right)<+\infty$ if and only if $\left\{A_{1}, \cdots, A_{n}\right\}$ is locally linearly regular at $a$.

We will provide necessary and/or sufficient conditions for the local linear regularity and establish formulas for the modulus $\eta\left(A_{1}, \cdots, A_{n} ; a\right)$. Let $\tau, \delta \in(0,+\infty)$. For convenience of presenting our results, we list the following inclusions.

$$
\begin{array}{ll}
\hat{N}(A, u) \cap B_{X^{*}} \subset \tau \sum_{i=1}^{n} N\left(A_{i}, u\right) \cap B_{X^{*}} & \forall u \in A \cap B(a, \delta), \\
\hat{N}(A, u) \cap B_{X^{*}} \subset \tau \sum_{i=1}^{n} N_{c}\left(A_{i}, u\right) \cap B_{X^{*}} \quad \forall u \in A \cap B(a, \delta), \\
\hat{N}(A, u) \cap B_{X^{*}} \subset \tau \sum_{i=1}^{n} \hat{N}\left(A_{i}, u\right) \cap B_{X^{*}} \quad \forall u \in A \cap B(a, \delta), \\
N(A, u) \cap B_{X^{*}} \subset \tau \sum_{i=1}^{n} N\left(A_{i}, u\right) \cap B_{X^{*}} \quad \forall u \in A \cap B(a, \delta), \\
N_{c}(A, u) \cap B_{X^{*}} \subset \tau \sum_{i=1}^{n} N_{c}\left(A_{i}, u\right) \cap B_{X^{*}} \quad \forall u \in A \cap B(a, \delta) .
\end{array}
$$

In terms of these inclusions, we define the following quantities (here $f, l$ and $c$ indicate the Fréchet, limiting and Clarke normal cones respectively):

$$
\begin{aligned}
& \beta_{l}^{f}(\delta):=\inf \{\tau>0 ;(4.5) \text { holds }\}, \quad \beta_{c}^{f}(\delta):=\inf \{\tau>0: \text { (4.6) holds }\} \\
& \beta_{f}(\delta):=\inf \{\tau>0 ;(4.7) \text { holds }\}, \quad \beta_{l}(\delta):=\inf \{\tau>0: \text { (4.8) holds }\}
\end{aligned}
$$

and $\quad \beta_{c}(\delta):=\inf \{\tau>0:(4.9)$ holds $\}$.

Then

$$
\beta_{l}^{f}(\delta) \leq \beta_{l}(\delta) \text { and } \beta_{c}^{f}(\delta) \leq \beta_{c}(\delta) \quad \forall \delta \in(0,+\infty)
$$

First we consider the case when $X$ is an Asplund space.

TheOrem 4.1. Let $X$ be an Asplund space. Then

$$
\lim _{\delta \rightarrow 0^{+}} \beta_{l}^{f}(\delta) \leq \eta\left(A_{1}, \cdots, A_{n} ; a\right) .
$$

Consequently, if $\left\{A_{1}, \cdots, A_{n}\right\}$ is locally linearly regular at a then there exist $\tau, \delta \in$ $(0,+\infty)$ such that (4.5) holds.

Proof. If $\eta\left(A_{1}, \cdots, A_{n} ; a\right)=+\infty$, then (4.11) trivially holds. Next assume that $\eta\left(A_{1}, \cdots, A_{n} ; a\right)<\infty$. Let $\tau \in\left(\eta\left(A_{1}, \cdots, A_{n} ; a\right),+\infty\right)$. Then there exists $\delta_{0}>0$ such that

$$
d(x, A) \leq \tau \sum_{i=1}^{n} d\left(x, A_{i}\right) \quad \forall x \in B\left(a, \delta_{0}\right) .
$$


Let $u \in A \cap B\left(a, \frac{\delta_{0}}{2}\right)$ and $x^{*} \in \hat{N}(A, u) \cap B_{X^{*}}$. Let $k$ be an arbitrary natural number. Then there exists $r \in\left(0, \frac{\delta_{0}}{2}\right)$ such that (2.5) holds with $\varepsilon=\frac{1}{k}$. Noting that $B(u, r) \subset$ $B\left(a, \delta_{0}\right)$, it follows from (4.12) that

$$
\left\langle x^{*}, x-u\right\rangle \leq \tau \sum_{i=1}^{n} d\left(x, A_{i}\right)+\frac{1}{k}\|x-u\| \quad \forall x \in B(u, r) .
$$

This and [17, Theorem 2.33] imply that there exist $u_{k, i} \in A_{i}, u_{k, i}^{*} \in X^{*}$ and $x_{k, i}^{*} \in$ $\frac{1}{k} B_{X^{*}}(i=1, \cdots, n)$ such that

$$
\begin{gathered}
\left\|u_{k, i}-u\right\|<\frac{1}{k}, \quad u_{k, i}^{*} \in \hat{\partial} d\left(\cdot, A_{i}\right)\left(u_{k, i}\right) \subset \hat{N}\left(A_{i}, u_{k, i}\right) \cap B_{X^{*}} \text { and } \\
\left\|x^{*}-\tau \sum_{i=1}^{n} u_{k, i}^{*}-x_{k, i}^{*}\right\|<\frac{1}{k} .
\end{gathered}
$$

Hence $\tau \sum_{i=1}^{n} u_{k, i}^{*} \stackrel{w^{*}}{\longrightarrow} x^{*}$ as $k \rightarrow \infty$. Since $B_{X^{*}}$ is sequentially compact with respect to the weak* topology, without loss of generality we assume $u_{k, i}^{*} \stackrel{w^{*}}{\longrightarrow} u_{i}^{*} \in N\left(A_{i}, u\right) \cap B_{X^{*}}$ as $k \rightarrow \infty$ (passing to a subsequence if necessary). Hence

$$
x^{*}=\tau \sum_{i=1}^{n} u_{i}^{*} \in \tau \sum_{i=1}^{n} N\left(A_{i}, u\right) \cap B_{X^{*}} .
$$

This shows that (4.5) holds for any $\delta \in\left(0, \frac{\delta_{0}}{2}\right)$, and so $\lim _{\delta \rightarrow 0^{+}} \beta_{l}^{f}(\delta) \leq \tau$. Letting $\tau \rightarrow \eta\left(A_{1}, \cdots, A_{n} ; a\right)$, it follows that (4.12) holds. The proof is completed.

Under the subsmoothness assumption of each $A_{i}$ at $a$, the following theorem shows that the equality in Theorem 4.1 holds and the necessary condition is also sufficient.

THEOREM 4.2. Let $X$ be an Asplund space and suppose that each $A_{i}$ is subsmooth at $a \in A$. Then

$$
\lim _{\delta \rightarrow 0^{+}} \beta_{l}^{f}(\delta)=\eta\left(A_{1}, \cdots, A_{n} ; a\right) .
$$

Consequently, $\left\{A_{1}, \cdots, A_{n}\right\}$ is locally linearly regular at a if and only if there exist $\tau, \delta \in(0,+\infty)$ such that (4.5) holds.

Proof. By Theorem 4.1, we need only show that

$$
\lim _{\delta \rightarrow 0^{+}} \beta_{l}^{f}(\delta) \geq \eta\left(A_{1}, \cdots, A_{n} ; a\right) .
$$

Since (4.13) trivially holds if $\lim _{\delta \rightarrow 0^{+}} \beta_{l}^{f}(\delta)=+\infty$, next we assume that $\lim _{\delta \rightarrow 0^{+}} \beta_{l}^{f}(\delta)<$ $+\infty$. Take an arbitrary $\tau \in\left(\lim _{\delta \rightarrow 0^{+}} \beta_{l}^{f}(\delta),+\infty\right)$ and $\delta>0$ such that (4.5) holds. Consider any $\varepsilon \in\left(0, \frac{1}{2}\right)$. Since each $A_{i}$ is subsmooth at $a$, Proposition 2.1 implies there exists $\delta_{1} \in(0, \delta)$ such that

$$
\left\langle x_{i}^{*}, x_{i}-a_{i}\right\rangle \leq d\left(x_{i}, A_{i}\right)+\frac{\varepsilon}{n}\left\|x_{i}-a_{i}\right\| \text { for all } x_{i} \in B\left(a, \delta_{1}\right)
$$


whenever $a_{i} \in A_{i} \cap B\left(a, \delta_{1}\right), x_{i}^{*} \in N_{c}\left(A_{i}, a_{i}\right) \cap B_{X^{*}}$ and $i=1, \cdots, n$. Let $r:=\frac{\delta_{1}}{2}$ and $x \in B(a, r) \backslash A$. Then $d(x, A) \leq\|x-a\|<r$. Let $\gamma \in\left(\max \left\{\frac{d(x, A)}{r}, \tau \varepsilon\right\}, 1\right)$. By Theorem 3.1 (applied to case (AF)), there exist $z \in \operatorname{bd}(A)$ and $x^{*} \in \hat{N}(A, z)$ with $\left\|x^{*}\right\|=1$ such that

$$
\left\langle x^{*}, x-z\right\rangle \geq \gamma\|x-z\|
$$

and $\gamma\|x-z\| \leq d(x, A)$. Thus, $\|x-z\| \leq \frac{d(x, A)}{\gamma}<r$. Hence

$$
\|z-a\| \leq\|z-x\|+\|x-a\|<2 r=\delta_{1}<\delta .
$$

It follows from (4.5) that there exists $x_{i}^{*} \in N\left(A_{i}, z\right) \cap B_{X^{*}}$ such that $x^{*}=\tau \sum_{i=1}^{n} x_{i}^{*}$. By (4.14), one has

$$
\left\langle x^{*}, x-z\right\rangle=\tau \sum_{i=1}^{n}\left\langle x_{i}^{*}, x-z\right\rangle \leq \tau \sum_{i=1}^{n}\left(d\left(x, A_{i}\right)+\frac{\varepsilon}{n}\|x-z\|\right) .
$$

This and (4.15) imply that $(\gamma-\tau \varepsilon)\|x-z\| \leq \tau \sum_{i=1}^{n} d\left(x, A_{i}\right)$ and hence

$$
d(x, A) \leq \frac{\tau}{(\gamma-\tau \varepsilon)} \sum_{i=1}^{n} d\left(x, A_{i}\right)
$$

(because $z \in A$ ). Therefore, $\eta\left(A_{1}, \cdots, A_{n} ; a\right) \leq \frac{\tau}{(\gamma-\tau \varepsilon)}$. It follows that (4.13) holds by letting $\gamma \rightarrow 1^{-}, \varepsilon \rightarrow 0^{+}$and $\tau \rightarrow \lim _{\delta \rightarrow 0^{+}} \beta_{l}^{f}(\delta)$. This completes the proof.

The following proposition and Theorem 4.1 explain that under the local linear regularity assumption, the subsmoothness and Clarke regularity of each $A_{i}$ imply, in some sense, the subsmoothness and the Clarke regularity of the intersection $A$, respectively.

Proposition 4.2. Let $X$ be an Asplund space and $\lim _{\delta \rightarrow 0^{+}} \beta_{c}^{f}(\delta)<+\infty$. Suppose that each $A_{i}$ is regular in the Clarke sense at all points of $A$ close to a. Then $A$ is regular in the Clarke sense at all points of $A$ close to a. If, in addition, each $A_{i}$ is subsmooth at a, then $A$ is subsmooth at a.

Proof. Take $\delta>0$ such that each $A_{i}$ is is regular in the Clarke sense (resp. subsmooth) at each point of $A \cap B(a, \delta)$. Then

$$
N_{c}\left(A_{i}, u\right)=\hat{N}\left(A_{i}, u\right) \quad \forall u \in A \cap B(a, \delta) .
$$

Considering smaller $\delta$ if necessary, one can find $\tau \in\left(\lim _{\delta \rightarrow 0^{+}} \beta_{c}^{f}(\delta),+\infty\right)$ such that that (4.5) holds. When each $A_{i}$ is subsmooth at $a$, it is easy to verify that for any $\varepsilon>0$ there exists $r \in(0, \delta)$ such that (2.3) holds whenever $u \in A \cap B(z, r)$ and $u^{*} \in \hat{N}(A, u) \cap B_{X^{*}}$. Thus, we need only show that

$$
N_{c}(A, u)=\hat{N}(A, u) \quad \forall u \in A \cap B(a, \delta) .
$$


Let $u \in A \cap B(a, \delta)$ and $u^{*} \in N(A, u)$. By (2.1), there exists a sequence $\left\{\left(u_{k}, u_{k}^{*}\right)\right\}$ in $(A \cap B(a, \delta)) \times X^{*}$ such that $u_{k} \rightarrow u$ and $u_{k}^{*} \stackrel{w^{*}}{\rightarrow} u^{*}$ with $u_{k}^{*} \in \hat{N}\left(A, u_{k}\right)$ for all $k$. It follows that $\left\{u_{k}^{*}\right\}$ is bounded. Without loss of generality, we assume that each $u_{k}^{*} \in B_{X^{*}}$. By (4.5) and (4.16), there exist $u_{k}^{*}(i) \in \hat{N}\left(A_{i}, u_{k}\right) \cap B_{X^{*}}$ such that $u_{k}^{*}=\tau \sum_{i=1}^{n} u_{k}^{*}(i)$. Without loss of generality, we can assume that $u_{k}^{*}(i) \stackrel{w^{*}}{\rightarrow} u^{*}(i) \in$ $N\left(A_{i}, u\right) \cap B_{X^{*}}, i=1, \cdots, n$ (passing to subsequences if necessary). Hence, $u^{*}=$ $\tau \sum_{i=1}^{n} u^{*}(i)$. On the other hand, by the definition of the Fréchet normal cone it is easy to verify that

$$
\sum_{i=1}^{n} \hat{N}\left(A_{i}, u\right) \subset \hat{N}(A, u) \quad \forall u \in A .
$$

It follows from (4.16) that $u^{*} \in \hat{N}(A, u)$. Therefore, $N(A, u) \subset \hat{N}(A, u)$, and so $N(A, u)=\hat{N}(A, u)$. Since $\hat{N}(A, u)$ is a convex cone, it follows from (2.1) that $N_{c}(A, u)=\operatorname{cl}^{*}(\hat{N}(A, u))$. This and Lemma 2.1 imply that (4.17) holds. The proof is completed.

Theorem 4.3. Let $X$ be an Asplund space. Suppose that each $A_{i}$ is subsmooth at $a$ and that each $A_{i}$ is regular in the Clarke sense at each point of $A$ close to a. Then,

(4.18) $\eta\left(A_{1}, \cdots, A_{n} ; a\right)=\lim _{\delta \rightarrow 0^{+}} \beta_{c}(\delta)=\lim _{\delta \rightarrow 0^{+}} \beta_{l}(\delta)=\lim _{\delta \rightarrow 0^{+}} \beta_{f}(\delta)=\lim _{\delta \rightarrow 0^{+}} \beta_{c}^{f}(\delta)$.

Proof. Since each $A_{i}$ is regular in the Clarke sense at each point of $A$ close to $a$, there exists $\delta>0$ such that (4.16) holds. Hence

$$
\lim _{\delta \rightarrow 0^{+}} \beta_{c}(\delta) \geq \lim _{\delta \rightarrow 0^{+}} \beta_{l}(\delta) \geq \lim _{\delta \rightarrow 0^{+}} \beta_{f}(\delta)=\lim _{\delta \rightarrow 0^{+}} \beta_{l}^{f}(\delta)=\lim _{\delta \rightarrow 0^{+}} \beta_{c}^{f}(\delta) .
$$

It follows that (4.18) holds if $\lim _{\delta \rightarrow 0^{+}} \beta_{l}^{f}(\delta)=+\infty$. Next assume that $\lim _{\delta \rightarrow 0^{+}} \beta_{l}^{f}(\delta)<+\infty$. By Proposition 4.2, $N_{c}(A, u)=N(A, u)=\hat{N}(A, u)$ for all $u \in A$ close to $a$. It follows from (4.16) and Theorem 4.2 that (4.18) holds. The proof is completed.

The following corollary is immediate from Theorem 4.3.

Corollary 4.1. Let $X$ be an Asplund space. Suppose that each $A_{i}$ is subsmooth at $a$ and that each $A_{i}$ is regular in the Clarke sense at each point of $A$ close to a. Then the following statements are equivalent.

(i) $\left\{A_{1}, \cdots, A_{n}\right\}$ is locally linearly regular at a.

(ii) There exist $\tau, \delta \in(0,+\infty)$ such that any one of (4.5)-(4.9) holds.

(iii) There exist $\tau, \delta \in(0,+\infty)$ such that (4.5) holds.

(iv) There exist $\tau, \delta \in(0,+\infty)$ such that such that

$$
\tilde{N}(A, u)=\sum_{i=1}^{n} \tilde{N}(A, u) \quad \forall u \in A \cap B(a, \delta)
$$


and

$$
\inf \left\{\sum_{i=1}^{n}\left\|x_{i}^{*}\right\|: \sum_{i=1}^{n} x_{i}^{*}=x^{*} \text { and } x_{i}^{*} \in \tilde{N}\left(A_{i}, u\right)\right\} \leq \tau\left\|x^{*}\right\|
$$

for any $u \in A \cap B(a, \delta)$ and $x^{*} \in \tilde{N}(A, u)$, where $\tilde{N}$ is any one of the Fréchet, limiting or Clarke normal cones.

The following corollary is immediate from Proposition 2.2 and Theorem 4.3

Corollary 4.2. Let $X$ be an Asplund space, $Y$ a Banach space and $f_{i}: X \rightarrow Y$ a continuously differentiable function $(i=1, \cdots, n)$. Let $C_{i}$ be a closed convex subset of $Y$ and $A_{i}:=f_{i}^{-1}\left(C_{i}\right)(i=1, \cdots, n)$. Let $a \in A$ and suppose that each $f_{i}^{\prime}(a)$ is surjective. Then (4.18) holds and (i) - (iv) in Corollary 4.1 are equivalent.

Next we consider the case when $X$ is a general Banach space.

Similar to the proof of Theorem 4.1 but with [8, Proposition 2.3.3] replacing [17, Theorem 2.33], one can prove the following result (indeed it is simpler because every Clarke normal cone is weak $^{*}$ closed and [8, Proposition 2.3.3], different from [17, Theorem 2.33], is an exact sum rule).

Proposition 4.3. Let $X$ be a Banach space. Then

$$
\lim _{\delta \rightarrow 0^{+}} \beta_{c}^{f}(\delta) \leq \eta\left(A_{1}, \cdots, A_{n} ; a\right) .
$$

Consequently, if $\left\{A_{1}, \cdots, A_{n}\right\}$ is locally linearly regular at a then there exist $\tau, \delta \in$ $(0,+\infty)$ such that (4.6) holds.

Similar to the proof of Theorem 4.2 but applying Theorem 3.1 to case (BC), we can prove the following proposition.

Proposition 4.4. Let $X$ be a Banach space and suppose that each $A_{i}$ be subsmooth at $a$. Suppose that there exist $\tau, \delta \in(0,+\infty)$ such that (4.9) holds. Then $\left\{A_{1}, \cdots, A_{n}\right\}$ is locally linearly regular at a and

$$
\eta\left(A_{1}, \cdots, A_{n} ; a\right) \leq \lim _{\delta \rightarrow 0^{+}} \beta_{c}(\delta) .
$$

The following example explains that if the subsmoothness assumption is dropped then Proposition 4.4 is not true even when $X$ is finite dimensional.

Example. Let $X=R^{2}, A_{1}=\left\{(s, t) \in R^{2}: s t \leq 0\right\}$ and

$$
A_{2}=\left\{(s, t) \in R_{+}^{2}:(s-1)^{2}+t^{2} \leq 1 \text { and } s^{2}+(t-1)^{2} \leq 1\right\} .
$$

By the definition of the Clarke tangent cone, it is easy to verify that $T_{c}\left(A_{1},(0,0)\right)=$ $\{(0,0)\}$. This means that $N_{c}\left(A_{1},(0,0)\right)=X^{*}$. Hence

$$
N_{c}\left(A_{1} \cap A_{2},(0,0)\right) \cap B_{X^{*}} \subset N_{c}\left(A_{1},(0,0)\right) \cap B_{X^{*}}+N_{c}\left(A_{2},(0,0)\right) \cap B_{X^{*}} .
$$


On the other hand, for every natural number $k$, let $x_{k}=\left(\frac{1}{k},\left(\frac{2}{k}-\frac{1}{k^{2}}\right)^{\frac{1}{2}}\right)$. Noting that $A_{1} \cap A_{2}=\{(0,0)\}$, it is easy to verify that

$$
d\left(x_{k}, A_{1} \cap A_{2}\right)=\left(\frac{2}{k}\right)^{\frac{1}{2}}, d\left(x_{k}, A_{1}\right)=\frac{1}{k} \text { and } d\left(x_{k}, A_{2}\right)=0 .
$$

Hence, $\frac{d\left(x_{k}, A_{1} \cap A_{2}\right)}{d\left(x_{k}, A_{1}\right)+d\left(x_{k}, A_{2}\right)}=(2 k)^{\frac{1}{2}} \rightarrow+\infty$. This shows that $\left\{A_{1}, A_{2}\right\}$ is not locally linearly regular at $(0,0)$.

Noting that (4.9) and (4.16) imply $N_{c}(A, u)=\hat{N}(A, u)$ for all $u \in A \cap B(a, \delta)$, similar to the proof of Theorem 4.3 we have the following result.

Proposition 4.5. Let $X$ be a Banach space. Suppose that each $A_{i}$ be subsmooth at $a$ and that each $A_{i}$ is regular in the Clarke sense at all points of $A$ close to a. Further suppose that there exist $\tau, \delta \in(0,+\infty)$ such that (4.9) holds. Then (4.18) holds.

Recently, Kruger [13, 14] studied a different kind of regularity of $\left\{A_{1}, \cdots, A_{n}\right\}$ at $a$ defined by $0<\lim _{\rho \rightarrow 0^{+}} \frac{1}{\rho} \sup \left\{r \geq 0: \bigcap_{i=1}^{n}\left(A_{i}-a_{i}\right) \cap\left(a+\rho B_{X}\right) \neq \emptyset, \forall a_{i} \in r B_{X}\right\}$.

Acknowledgments. The authors wish to thank the referees for careful reading of the paper and many valuable comments, which help to improve our presentation.

\section{REFERENCES}

[1] D. Aussel, A. Daniilidis and L. Thibault, Subsmooth sets: functional characterizations and related concepts, Trans. Amer. Math. Soc., 357 (2005), pp.1275-1301.

[2] A. Bakan, F. Deutsch and W. Li, Strong CHIP, normality, and linear regularity of convex sets, Trans. Amer. Math. Soc., 357 (2005), pp.3831-3863.

[3] H. H. Bauschke and J. M. Borwein, On projection algorithms for solving convex feasibility problems, SIAM Rev., 38 (1996), pp.367-426.

[4] H. H. Bauschke, J. M. Borwein and W. Li, Strong conical hull intersection property, bounded linear regularity, Jameson's property $(\mathrm{G})$, and error bounds in convex optimzation, Math. Program., 86 (1999), pp.135-160.

[5] H. H. Bauschke, J. M. Borwein and P. Tseng, Bounded linear regularity, strong CHIP, and CHIP are distinct properties, J. Convex Anal., 7 (2000), pp.395-413.

[6] F. Bernard and L. Thilbaut, Uniform prox-regularity of functions and epigraphs in Hilbert spaces, Nonlinear Anal., 60 (2005), pp.187-207.

[7] F. Bernard and L. Thilbaut, Prox-regularity of functions and sets in Banach spaces, Set-Valued Anal., 12 (2004), pp.25-47.

[8] F. H. Clarke, Optimization and Nonsmooth Analysis, Wiley, New York, 1983.

[9] F. H. Clarke, R. Stern and P. Wolenski, Proximal smoothness and the lower-C ${ }^{2}$ property, J. Convex Anal., 2 (1995), pp.117-144.

[10] F. Deutsch, W. Li and J. Swetits, Fenchel duality and the strong conicial hull intersection property, J. Optim. Theory Appl., 102 (1999), pp.681-695.

[11] F. Deutsch, W. Li and J. D. Ward, Best approximation from the intersection of a closed convex set and a polyhedron in Hilbert spaces, weak Slater conditions, and the strong conicial hull intersection property, SIAM J. Optim., 10 (2000), pp.252-268.

[12] G. Jameson, The duality of pair of wedges, Proc. Lond. Math. Soc., 24 (1972), pp.531-547. 
[13] A. Y. Kruger, Stationary and regularity of set systems, Pac. J. Optim., 1 (2005), pp.101-126.

[14] A. Y. Kruger, About regularity of collection of sets, Set-valued Anal., 14 (2006), pp.187-206.

[15] A. S. Lewis and J. S. Pang, Error bounds for convex inequality systems, In: Crouzeix, J. P., ed., Generalized Convexity, Proceedings of the Fifth Sysposium on Generalized Convexity, Luminy Marseille, 1997, pp.75-10.

[16] C. Li and K. F. Ng, Constraint qualification, the strong CHIP, and best approximation with constraints in Banach spaces, SIAM J. Optim., 14(2003), pp.584-607.

[17] B. S. Mordukhovich, Variational Analysis and Generalized differentiation I/II, Springer-verlag, Berlin Heidelberg, 2006.

[18] B. S. Mordukhovich and Y. Shao, Nonsmooth sequential analysis in Asplund spaces, Trans. Amer.Math. Soc., 348 (1996), pp. 1235-1280.

[19] K. F. Ng and W. H. Yang, Error bounds for abstract linear inequality systems, SIAM J. Optim., 13 (2002), pp.24-43.

[20] K. F. Ng and W. H. Yang, Regularities and relations to error bounds, Math. Program., 99 (2004), pp.521-538.

[21] J. S. Pang, Error bounds in mathematical programming, Math. Program., 79 (1997), pp.299332.

[22] R. R. Phelps, Convex functions, Monotone Operators and Differentiability, Lecture Notes in Math., Vol. 1364, Springer, New York, 1989.

[23] R. Poliquin, R. T. Rockafellar and L. Thibault, Local differentiability of distance functions, Trans. Amer. Math. Soc., 352 (2000), pp.5231-5249.

[24] R. T. Rockafellar and R. J.-B. Wets, Variational Analysis, Springer-Verlag, Berlin, 1998.

[25] X. Y. Zheng and K. F. Ng, Metric regularity and constraint qualifications for convex inequalities on Banach spaces, SIAM J. Optim., 14(2003), pp.757-772. 